\title{
Response to conventional nutritional treatment of severely malnourished hospitalized children in the context of HIV infection at Yekatit 12 hospital, Addis Ababa, Ethiopia
}

\author{
Tesfaye Taye Gelaw ${ }^{1,}$, Amha Mekasha Wondemagegn ${ }^{2}$ \\ ${ }^{1}$ Department of Pediatrics and child health, Bahir Dar University, Bahir Dar, Ethiopia \\ ${ }^{2}$ Department of Pediatrics and child health, AAU, Addis Ababa, Ethiopia
}

\section{Email address:}

testaye2006@yahoo.com (T. T. Gelaw), amekashaw@yahoo.com (A. M. Wondemagegn)

\section{To cite this article:}

Tesfaye Taye Gelaw, Amha Mekasha Wondemagegn. Response to Conventional Nutritional Treatment of Severely Malnourished Hospitalized Children in the Context of HIV Infection at Yekatit 12 Hospital, Addis Ababa, Ethiopia. Science Journal of Clinical Medicine. Vol. 2, No. 6, 2013, pp. 176-182. doi: 10.11648/j.sjcm.20130206.16

\begin{abstract}
Background: The aim of this study was to describe the response to nutritional treatment of severely malnourished hospitalized children in relation to HIV infection Methods: The study was conducted in the paediatric wards of Yekatit 12 hospital, which is situated in Addis Ababa, Ethiopia serving as a referral hospital for clinics, health centers and hospitals situated in and out of Addis Ababa. It also serves as a teaching hospital for AAU Medical students and Residents. We studied 300 severely malnourished children (presence of oedema, weight for age $<60 \%$ on $\mathrm{HC}$ and/ or weight-for-height $<70 \%$ ). At admission, HIV serology was confirmed by ELISA, for children $>18$ months of age, and PCR (DBS) was performed for those $<18$ months and CD4+ cells were measured for those who turned out to be positive. Complete blood count (CBC), including differential counts, was determined using a Coulter counter and/or manually. The study is a prospective observational analytic cohort study. Result: Of the 300 children, 75 were HIV Positive (cases) and the remaining 225 were controls (1:3 ratio). There was no significant difference by sex, age group, site of residency, family in come, family educational status, medical illness, hematologic profiles, immune category and presence or absence of edema in the treatment out come of HIV infected children compared to uninfected once. Among HIV infected forty three (43/75) failed to respond to the nutritional intervention when compared to those who are not infected $(225 / 38)$ (Adjusted OR $=6.61,95 \% \mathrm{CI}=0.053$, 0.423). Conclusion: HIV infection is a major challenge in addressing nutritional intervention for those children who are severely malnourished admitted in Yekatit 12 hospital. An intervention to alleviate the challenge in managing the problem has to be designed based on further interventional studies.
\end{abstract}

Keywords: HIV, Malnutrition, Treatment Response

\section{Background}

Now a days, malnutrition is a significant problem in HIV infected children and studies are showing that the prevalence of malnutrition is estimated to be around $26 \%^{(1)}$ in the HIV infected population compared to approximately $9 \%$ of children below 5 years of age suffer from wasting (weight-for-height below - 3 standard deviations (- 3 SD) of the National Center for Health Statistics (NCHS)/WHO reference values) ${ }^{(17)}$.

HIV/AIDS is a serious public health problem due to its health, economic, psychologic and social consequences.
Among affected individual, malnutrition is common in the advanced stages of the disease. Nutritional intervention should be applied early and be individualized, with periodic and constant screening ${ }^{(7)}$.

Routine nutritional assessment should be performed at every clinical visit to intervene before they acquire the severe form of malnutrition, because of their poor response to nutritional treatment. A more extensive evaluation may be required for children with growth failure or other evidence of malnutrition ${ }^{(8)}$.

A study undertaken in a central nutritional rehabilitation 
unit in southern Malawi to assess the impact of HIV infection on clinical presentation and case fatality rate shows HIV seroprevalence in 250 severely malnourished children over 1 year of age to be $34.4 \%$ and overall mortality $28 \%$ \& HIV infection was associated significantly more frequently with marasmus $(62.2 \%)$ than with kwashiorkor $(21.7 \%)(\mathrm{P}<$ $0.0001)^{(9)}$.These observations appear to imply that the development of oedema requires a certain degree of immunocompetence, which is an interesting clue to the patho-physiology of oedema in severe malnutrition. Generally speaking, Severe protein energy malnutrition is associated with the depletion of the haematological \& lymphocyte subsets, \& this depletion is exacerbated by the presence of HIV-1 infection. Cell mediated immuno-suppression is more marked in non-oedematous severe malnutrition, regardless of the HIV status (10). Mortality risk among severely malnourished children was significantly increased among those children with HIV \& was associated with a lower $\mathrm{CD} 4 \%{ }^{(4)}$.

Responsiveness to nutritional interventions in people living with HIV/AIDS (PLWHA) depends on viral load, stage of disease, concurrent treatment, nutritional status, and presence or absence of opportunistic infections (OIs). However, there are few data on the direct effect of dietary intake on these variables in HIV-positive people, particularly in developing countries ${ }^{(2)}$. According to WHO, the effect of nutritional intervention on severely malnourished HIV infected children is not well evaluated and it is a research gap that has to be filled. ${ }^{(3)}$

Currently, exactly the same protocol is used in HIV positive and negative patients even though the mutually reinforcing relationship between HIV and malnutrition is little understood and has complicated the treatment of severe malnutrition, Challenging more traditional approaches and protocols ${ }^{(4)}$. According to the protocol for management of severe acute malnutrition, FMOH ETHIOPIA, 2007, they respond well to the treatment regimen, usually regaining their appetites and gaining weight at the same rate as HIV negative patients ${ }^{(5)}$. But Information about the pattern of response to nutritional therapy of severely malnourished HIV infected children is limited in Ethiopia. Hence, we need to evaluate the effect of our nutritional intervention on HIV infected severely malnourished children who are being treated with same protocol as that of HIV negative severely malnourished children.

\section{Methods and Materials}

\subsection{Study Setting}

Yekatit 12 hospital - is located in Addis Ababa; Ethiopia. It provides a health care service at an out patient and in patient level as a referral centre for health centers and hospitals in Addis Ababa as well as different regions of the country. The Hospital was using the FMOH guide line for the management of severe acute malnutrition adapted from UNICEF.

\subsection{Study Design and Subject Selection}

The study design is a prospective observational analytic cohort study. The study period spanned from November $1 / 2008$ to August 17/2009. The admissions for nutritional therapy to Yekatit 12 Hospital, over this time period, are included in this study. All severely malnourished children below the age of 144 months attending the department of Paediatrics and Child health between November 1, 2008 and August 17, 2009, were screened for severe malnutrition, using weight, height (or length for children less than 2 years) and presence of oedema. Measurements were taken in accordance with WHO standard techniques and compared with National Centre for Health Statistics (NCHS) reference population. Severe malnutrition was defined as weight for age $<60 \%$ on Harvard curve, weight-for height of $<-3$ zscore and/or presence of oedema.

Exclusion criteria:

1. Length $<49 \mathrm{~cm}$; since the NCHS reference does not provide reference values.

2. Children who are preterm at delivery \& aged $<2 \mathrm{yrs}$; because catch up growth will not be completed before this age.

3. Children whose family are not volunteer their child to be screened.

4. Children whose caregivers didn't agreed to join the study.

5. Children died before the 4th day of nutritional therapy.

A total of 300 children were screened during the period. Of these, 75 (25\%) were HIV infected and the rest 225(75\%) are HIV negative (3:1 ratio).

Their caregivers were informed of the study objectives and methods to be used, and requested for their agreement before filling the questionnaire. They were also counseled on having the HIV status of their children determined. Medical doctors (Interns and Residents) collected the children's demographic characteristics by interviewing the caregiver, filling in questionnaire, and determining their health characteristics using clinical history, physical examination and laboratory examinations.

A checklist was used to collect additional information from the patient's file immediately after the post-admission round by a team of doctors.

The caregivers received pre-test and post-test counseling for HIV testing by a trained and experienced counselor.

Caregivers of children who tested positive for HIV were counseled, and advised to attend the Paediatric Infectious Disease Clinic, a specialized out patient clinic at the department for HIV positive children, for further management and subsequent follow up. The clinic offers comprehensive HIV/AIDS care including free antiretroviral drugs.

All enrolled children were followed-up daily by a group of physicians on their respective wards who recorded the individual child's health development on multi- chart. 


\subsection{Sample Size and Selection $(15,16)$}

With the assumption of $50 \% \quad\left(\pi_{1}\right)$ of severely malnourished HIV negative children and 30\% $\left(\pi_{2}\right)$ of severely malnourished HIV positive children will respond to optimal nutritional treatment; level of significance $5 \%(\alpha)$; non-respondent rate $10 \%$, and the power of the study taken as $80 \%(1-\beta)$. With this the sample size is calculated to be 300 . This was calculated with the help of statistical software EPI INFO. For every severely malnourished HIV infected cases the next three consecutive severely malnourished HIV negative children who are put on treatment for severe malnutrition are taken.

\subsubsection{Laboratory Investigations}

- Haematological tests - were done by coulter machine \& manually.

- Total serum protein and urinalysis- for those having edematous malnutrition.

- $\quad$ HIV testing -

- HIV serology test was used.

Criteria

Primary failure to respond:

Failure to regain appetite

Failure to start to lose oedema

Oedema still present

Failure to gain at least $5 \mathrm{~g} / \mathrm{kg}$ of body weight per day

Secondary failure to respond:

Failure to gain at least $5 \mathrm{~g} / \mathrm{kg}$ of body weight per day for 3 successive days

\subsubsection{Nutritional Treatment Failure}

1. Failure to achieve target weight up to 6 weeks $(\mathrm{Wt} / \mathrm{Ht}=85 \%)$

2. Death at any time during nutritional rehabilitation after 4th day of admission.

\subsubsection{Nutritional Treatment Response}

1. Target weight achievement $(85 \%=\mathrm{wt} / \mathrm{Ht})$ within 4 weeks of nutritional treatment and/or within $4-6$ weeks

2. MUAC $>=12 \mathrm{~cm}$ (if length $>=75 \mathrm{~cm})^{(22)}$.

\subsubsection{Data Collection/Implementation}

Data were taken in two phases; first immediately after admission by taking history, doing physical examination and investigations. Secondly nutritional treatment registry (multi chart) is followed and revised for completeness at the time of discharge.

\subsubsection{Data Analysis/Management}

Raw data was cross-checked for completeness and correct labeling, and arranged and stored securely accessible only to the researcher. Stored data included patient identification; inpatient or hospital number and study code. The data was analyzed using SPSS version 17.
- $\quad$ DBS (DNA PCR) was performed to confirm positive EIA test for children $<18$ months.

- The CD4+ cells determined for those who turned out to be positive.

\subsubsection{Operational Definitions}

Severe malnutrition ${ }^{(19,20)}$ - It is severe malnutrition if the wasting is severe $(\mathrm{W} / \mathrm{H}<70 \%$ NCHS median or a low MUAC), WFA $<60 \%$ on Harvard curve for under fives or there is oedema. Oedematous cases are always classified as severe.

HIV disease - A rational approach to diagnosis of paediatric HIV infection requires a combination of clinical finding and laboratory-support. HIV-specific laboratory tests provide a definitive diagnosis, add to the strength of a clinical diagnosis (e.g., by confirming exposure), or can actively aid the exclusion of HIV disease ${ }^{(21)}$. We use laboratory supported approach to diagnose HIV disease.

\subsubsection{Nutritional Treatment Failure ${ }^{(17)}$}

Time after admission

Day 4

Day 4

Day 10

Day 10

\section{During rehabilitation}

\subsubsection{Ethical Considerations}

Ethical clearance was obtained at three levels in accordance with the rules and regulation of the university (Department Research and Publication Committee, Faculty Research and Publication Committee and the University Research and Publication Office).

\section{Results}

Three hundred (300) severely malnourished children aged from 2 months to 144 months (225 were HIV negative, controls and 75 were HIV positive, cases; with an assumption of 3:1) were admitted to the pediatrics ward of Yekatit 12 Hospital during the time period November 1, 2008 - August 17, 2009 meeting the criteria and followed for their response to nutritional therapy. One hundred sixty seven (167) were males $(55.7 \%)$ of which $47(28 \%)$ were HIV positive and one hundred thirty three (133) were females (44.3\%) of which $28(21 \%)$ were HIV positive.

The mean age of children subjected to the study was twenty two (22.26 months), the median being fifteen (15) months. One hundred fourteen (114) were less than 12 months of age, of which 25 (22\%) are HIV positive; one hundred thirty eight (138) are between the age of 12 months \& 35 months, of which 26 (18.8\%) are HIV positive; twenty 
three (23) are between the ages of 36 months and 59 months, of which $9(39 \%)$ are HIV positive; \& 25 are between the ages of 60 months \& 144 months, of which 15 (60\%) are HIV positive.

Among the three hundred; 215 were from urban sites of which $68(31.6 \%)$ were HIV positive and 85 were from rural area of which $7(8 \%)$ were HIV positive.

Among one hundred fourteen (114) children who are less than twelve (12) months of age thirty nine (39) failed to respond to our nutritional therapy $(34.2 \%)$; Adjusted OR, $95 \% \mathrm{CI}=0.31(0.08,1.23)$. Of one hundred thirty eight $(138)$ children between the ages of twelve months \& thirty five months twenty six (26) failed to respond to the nutritional therapy $(18.8 \%)$; Adjusted OR, 95\% $\mathrm{CI}=0.51(0.14,1.94)$. Out of twenty three (23) children aged from thirty six (36) weeks to fifty nine (59) weeks nine (9) failed to respond for optimal nutritional therapy (39\%); Adjusted OR, 95\% $\mathrm{CI}=$ $0.28(0.06,1.41)$. And out of twenty five $(25)$ children aged from 60 to 144 months, seven (7) fail to respond (28\%).

Among one hundred sixty seven (167) male children forty four (44) failed to respond to optimal nutritional therapy $(26.4 \%)$ and out of one hundred thirty three (133) female children thirty seven (37) failed to respond too (27.8\%); Adjusted OR, 95\%CI $=0.74(0.39,1.41)$. Totally out of 300 children eighty one (81) failed to respond for the nutritional intervention (27\%).

Among two hundred fifteen (215) children from urban area seventy one (71) failed to respond to nutritional therapy (33\%) and out of eighty five (85) children from rural area ten (10) failed to respond (11.8\%). Adjusted OR, 95\%CI $=0.45$ $(0.18,1.17)$.

Of all the severely malnourished children seventy three (73) have pneumonia $(24.3 \%)$, thirty three (33) have tuberculosis $(11 \%)$, twenty eight (28) have sepsis $(9.3 \%)$, four (4) had UTI(1.3\%), sixty three (63) have diarrhea (21\%), fifty three (53) cases were with no identified associated medical problems $(17.7 \%) \&$ the remaining thirty two (32) have other problems in group (10.7\%). Tuberculosis and sepsis have an impact on treatment out come which is statistically significant. Adjusted OR, $95 \% \mathrm{CI}=0.22(0.05,0.93)$ and $0.22(0.05,0.90)$ respectively.

When we compare treatment out come in relation to their hematologic parameters, they have nothing to do with the nutritional treatment out come.

One hundred forty six (146) children were having edematous type of malnutrition out of which twenty six (26) were HIV positive $(17.8 \%)$. The remaining one hundred fifty four (154) children were having non edematous malnutrition of which forty nine (49) were HIV infected (31.8\%). HIV infected children are less likely to present with edema when compared to those who are not infected (OR, $95 \% \mathrm{CI}=0.464,(0.270,0.799))$.

Among the children who were infected with HIV forty three (43) failed to respond to optimal nutritional therapy $(57.3 \%)$ where as in those who are HIV negative only thirty eight(38) failed to respond for nutritional therapy(16.9\%). OR, 95\%CI $=0.18(0.09,0.40)$. Generally speaking nutritional intervention failure is significant in all phases of nutritional intervention in HIV infected children when compared to the HIV non infected children.

Among three hundred (300) severely malnourished children one hundred fifty four (154) were with edematous form and the remaining one hundred forty six (146) had the non edematous form. Whether being edematous malnutrition or non edematous malnutrition, the treatment response to nutritional treatment is not affected with $\mathrm{OR}$, $95 \% \mathrm{CI}=0.89(0.43,1.85)$.

The response to nutritional treatment is affected by WHO clinical stage and immune category; OR, 95\%CI $=3.62$ $(2.56,5.13)$ and $7.38(1.19,45.69)$ for stage IV and severe immune suppression respectively when compared to those who are not HIV infected.

Table 1. Treatment out come vs hiv status and other socio-demographic and clinical parameters.

\begin{tabular}{|c|c|c|c|c|c|}
\hline \multirow{2}{*}{\multicolumn{2}{|c|}{ Characteristics }} & \multicolumn{2}{|c|}{ Treatment out come } & \multirow{2}{*}{$\begin{array}{c}\text { Crude OR, } \\
95 \% \text { CI } \\
\end{array}$} & \multirow{2}{*}{$\begin{array}{c}\text { Adjusted OR, } \\
95 \% \text { CI } \\
\end{array}$} \\
\hline & & Failed & Improved & & \\
\hline \multirow{4}{*}{ Age in months } & $<12$ & 39 & 75 & $0.75(0.29,1.94)$ & $0.31(0.08,1.23)$ \\
\hline & $12-35$ & 26 & 112 & $1.68(0.63,4.42)$ & $0.51(0.14,1.94)$ \\
\hline & $36-59$ & 9 & 14 & $0.60(0.18,2.03)$ & $0.28(0.06,1.41)$ \\
\hline & $60-144$ & 7 & 18 & 1 & 1 \\
\hline \multirow{2}{*}{ Sex } & Male & 44 & 123 & 1 & 1 \\
\hline & Female & 37 & 96 & $0.93(0.56,1.55)$ & $0.74(0.39,1.41)$ \\
\hline \multirow{2}{*}{ Residency } & Urban & 71 & 144 & 1 & 1 \\
\hline & Rural & 10 & 75 & $3.70(1.80,7.59)$ & $0.45(0.18,1.17)$ \\
\hline \multirow{4}{*}{$\begin{array}{l}\text { Educational } \\
\text { Status, mother }\end{array}$} & Can't read/write & 22 & 118 & 1 & 1 \\
\hline & $\begin{array}{l}\text { Elementary } \\
\text { Complete }\end{array}$ & 15 & 45 & $0.57(0.27,1.20)$ & $0.84(0.29,2.46)$ \\
\hline & Secondary \& College & 22 & 25 & $0.22(0.10,0.45)$ & $0.61(0.15,2.46)$ \\
\hline & Orphan & 22 & 31 & $0.28(0.14,0.58)$ & $2.28(0.08,65.58)$ \\
\hline \multirow{4}{*}{$\begin{array}{l}\text { Educational } \\
\text { Status, father }\end{array}$} & Can't read/write & 9 & 64 & 1 & 1 \\
\hline & $\begin{array}{l}\text { Elementary } \\
\text { Complete }\end{array}$ & 17 & 73 & $0.60(0.25,1.45)$ & $1.07(0.34,3.36)$ \\
\hline & $\begin{array}{l}\text { Secondary \& } \\
\text { College }\end{array}$ & 33 & 51 & $0.22(0.09,0.50)$ & $0.64(0.14,2.98)$ \\
\hline & Orphan & 22 & 31 & $0.19(0.08,0.48)$ & $0.37(0.01,14.77)$ \\
\hline
\end{tabular}




\begin{tabular}{|c|c|c|c|c|c|}
\hline \multirow{2}{*}{\multicolumn{2}{|c|}{ Characteristics }} & \multicolumn{2}{|c|}{ Treatment out come } & \multirow{2}{*}{$\begin{array}{c}\text { Crude OR, } \\
95 \% \text { CI }\end{array}$} & \multirow{2}{*}{$\begin{array}{c}\text { Adjusted OR, } \\
95 \% \text { CI }\end{array}$} \\
\hline & & Failed & Improved & & \\
\hline \multirow{5}{*}{ Monthly income } & $<500$ & 19 & 77 & 1 & 1 \\
\hline & $500-1,000$ & 24 & 90 & $0.93(0.47,1.82)$ & $1.58(0.62,4.05)$ \\
\hline & $1,000-1,500$ & 11 & 16 & $0.36(0.14,0.89)$ & $1.29(0.29,5.73)$ \\
\hline & $>1,500$ & 6 & 6 & $0.25(0.07,0.85)$ & $1.02(0.17,6.21)$ \\
\hline & Orphan & 21 & 30 & $0.35(0.17,0.75)$ & $0.59(0.01,27.99)$ \\
\hline \multirow{2}{*}{ Pneumonia } & No & 60 & 167 & 1 & 1 \\
\hline & Yes & 21 & 52 & $0.89(0.49,1.60)$ & $0.44(0.13,1.53)$ \\
\hline \multirow{2}{*}{ Tuberculosis } & No & 70 & 197 & 1 & 1 \\
\hline & Yes & 11 & 22 & $0.71(0.33,1.54)$ & $0.22(0.05,0.93)$ \\
\hline \multirow{2}{*}{ OI's } & No & 71 & 214 & 1 & 1 \\
\hline & Yes & 9 & 5 & $0.19(0.06,0.58)$ & $0.23(0.04,1.32)$ \\
\hline \multirow{2}{*}{ Sepsis } & No & 66 & 206 & 1 & 1 \\
\hline & Yes & 15 & 13 & $0.28(0.13,0.61)$ & $0.22(0.05,0.90)$ \\
\hline \multirow{2}{*}{ Diarrhea } & No & 71 & 167 & 1 & 1 \\
\hline & Yes & 10 & 52 & $2.21(1.06,4.60)$ & $0.97(0.26,3.69)$ \\
\hline \multirow{2}{*}{$\begin{array}{l}\text { Associated } \\
\text { Illness }\end{array}$} & No & 10 & 43 & 1 & 1 \\
\hline & Yes & 71 & 176 & $0.58(0.28,1.21)$ & $1.38(0.36,5.25)$ \\
\hline \multirow{2}{*}{$\begin{array}{l}\text { Nutritional } \\
\text { Status }\end{array}$} & Non edematous & 48 & 106 & 1 & 1 \\
\hline & Edematous & 33 & 113 & $1.55(0.93,2.60)$ & $0.89(0.43,1.85)$ \\
\hline \multirow{2}{*}{ HIV status } & Negative & 38 & 187 & 1 & 1 \\
\hline & Positive & 43 & 32 & $0.15(0.08,0.27)$ & $0.18(0.09,0.40)$ \\
\hline \multirow{3}{*}{ Hematocrit } & Normal & 33 & 94 & 1 & 1 \\
\hline & Anemia & 46 & 112 & $0.86(0.51,1.44)$ & $0.70(0.36,1.38)$ \\
\hline & High Hematocrit & 2 & 13 & $2.28(0.49,10.6)$ & $2.91(0.46,18.34)$ \\
\hline \multirow{3}{*}{ WBC Count } & Normal & 60 & 170 & 1 & 1 \\
\hline & Leukopenia & 10 & 26 & $0.92(0.42,2.01)$ & $1.56(0.55,4.45)$ \\
\hline & Leukocytosis & 11 & 23 & $0.74(0.34,1.60)$ & $0.67(0.26,1.72)$ \\
\hline \multirow{3}{*}{ Platelet } & Normal & 49 & 154 & 1 & 1 \\
\hline & $<150,000$ & 19 & 29 & $0.49(0.25,0.94)$ & $0.93(0.38,2.27)$ \\
\hline & $>450,000$ & 13 & 36 & $0.88(0.43,1.79)$ & $1.05(0.44,2.51)$ \\
\hline
\end{tabular}

Table 2. Treatment outcome vs clinical stage and immune category when compared with non hiv infected.

\begin{tabular}{|c|c|c|c|c|}
\hline \multirow{2}{*}{\multicolumn{2}{|c|}{ Characterstics }} & \multicolumn{2}{|c|}{ Treatment Out Come } & \multirow{2}{*}{$\begin{array}{c}\text { OR, } \\
95 \% \text { CI }\end{array}$} \\
\hline & & Failed & Improved & \\
\hline \multirow{4}{*}{ WHO Clinical stage } & Stage I & - & - & - \\
\hline & Stage II & 0 & 1 & $0.00(0.00,87.63)$ \\
\hline & Stage III & 2 & 5 & $0.92(0.12,5.68)$ \\
\hline & Stage IV & 41 & 26 & $3.62(2.56,5.13)$ \\
\hline \multirow{4}{*}{ Immune category } & Non significant & 3 & 2 & $1.75(0.22,14.22)$ \\
\hline & Mild & 6 & 7 & $0.69(0.09,5.29)$ \\
\hline & Advanced & 13 & 6 & $1.21(0.18,8.12)$ \\
\hline & Severe & 21 & 17 & $7.38(1.19,45.69)$ \\
\hline
\end{tabular}

\section{Discussion}

In this prospective analytic cohort study, the main finding is that HIV infected severely malnourished children seem to respond less than HIV uninfected severely malnourished children to our nutritional intervention based on the guide line for severe acute malnutrition management, FMOH-ETHIOPIA. This result is in accordance with what is documented in the National guide line for management of severe acute malnutrition; FMOH- Ethiopia,(5) and in a research done in Burundi.(6) This implies that the effects of HIV/AIDS and malnutrition are inter related and exacerbate one another in a vicious cycle.(5)
Irrespective of the age, sex, residency, family income, educational status, associated medical illnes, and hematologic profiles the nutritional treatment out come seems solely significantly affected by the HIV status of the malnourished children. As stated by Kate Sadler, nutrition adviser for Valid International; high levels of poverty, food insecurity, childhood illness and HIV/AIDS combine to increase the risk of malnutrition, which is associated with more than $50 \%$ of childhood deaths in the developing world.(11) This contradiction might be due to the fact that the individuals included in the study are all in almost similar socio-economic status so that all are affected by these socio-demographic factors equally.

It is interesting to notice that the HIV-positive children 
less often present with oedema, that is, oedema was present in just about $34.7 \%$ of the HIV-positive children, while it was seen in about $53.3 \%$ of the HIV-negative children. This is statistically significant with $\mathrm{OR}, 95 \% \mathrm{CI}=0.464(0.270$, 0.799). This is consistent with a research done at Kampala, Uganda.(10) The possible explanation for the preference development of marasmus in HIV infected children than edematous malnutrition is the development of edema requires a certain degree of immuno competence.(10)

Those with specific infections, like tuberculosis, sepsis do have statistically significant impact on the nutritional treatment response compared to previous studies done that is In favor with the Report on Food and Nutrition for People Living with HIV/AIDS, The United States President's Emergency Plan for AIDS Relief, May 2006. Responsiveness to nutritional interventions in people living with HIV/AIDS (PLWHA) depends on viral load, stage of disease, level of immune suppression, concurrent medical treatment, nutritional status, and presence or absence of opportunistic infections (OIs).(23) Probably the children with OI's are very few in number which will retard us back from giving a conclusion so we recommend a further study on this specific topic. The finding that we have as to the response to nutritional treatment with regard to the clinical stage and immune category is similar to what is reported in a research done before and published on New England Journal of Medicine (23). Adjusted OR, 95\%CI = $3.62(2.56,5.13)$ and $7.38(1.19,45.69)$ respectively.

\section{Conclusion}

The response to nutritional treatment of HIV infected severely malnourished children is less when compared to those who are not HIV infected both being treated with the same protocol.

The response to nutritional intervention is dependent on:

- Clinical stage of HIV disease.

- Degree of immunosuppression.

Infections like tuberculosis and sepsis also affect the response to nutritional intervention.

\section{Recommendation}

We recommend the following:

A. In order to treat or prevent HIV-associated malnutrition:

1. The nutritional status \& history should be assessed in each HIV positive subject as early as possible before irreversible damage has happened.

2. Independent of the actual nutritional status, each patient should obtain a nutrition education as a preventive measure.

3. A continuous nutrition therapy becomes necessary in the case of evident or imminent malnutrition.

B. An intervention to alleviate the challenge in managing the problem has to be designed based on further interventional studies.

C. The effect of nutritional intervention on severely malnourished HIV infected children is not well evaluated and it is a research gap that has to be filled.

\section{Limitation of the Study}

This is a study done at a single hospital and most of the cases are from urban area. So it may not be extrapolated to represent the whole country because eighty five percent of the people of the country are living in the rural area where the actual magnitude of the problem is not well known.

\section{Acknowledgements}

We would like to extend our appreciation to the administration staff and health care workers of Yekatit 12 Hospital for their excellent cooperation. Our appreciation also goes to the Interns and Residents who were practicing at Yekatit 12 Hospital during the time period when we were collecting the data; without whom we were not able to accomplish the task.

\section{References}

[1] Susan Thurstans: HIV prevalence in severely malnourished children admitted to nutrition rehabilitation units in Malawi: Geographical \& seasonal variations a cross-sectional Study; BMC Pediatrics 2008, 8:22

[2] The USA President's emergency plan for AIDS relief, Report on food \& nutrition for PLWHA, May 2006.

[3] Stephen M. Arpadi, Growth failure in HIV infected children, WHO, department of nutrition for health \& development, 2005 .

[4] Action against hunger UK, Research: Developing Therapeutic Nutritional Care for HIV-Affected Children.

[5] FMOH-ETHIOPIA, Protocol for the management of severe acute malnutrition, 2007.

[6] Exceler JL: Malnutrition and HIV infection in children in a hospital milieu in Burundi: Pediatrie. 1987; 42:9:715-8.

[7] Fajardo-Rodriguez A, Lara del Rivero-Vera CM; Nutritional intervention in HIV/AIDS: practical guide for its implementation and follow-up; Gac Med Mex. 2001 Sep-Oct; 137: 5:489-500.

[8] The ICAP Pediatric clinical manual, 2005.

[9] Kessler L.; Daley H.; Malenga G.; Graham S. The impact of HIV type 1 on the management of severe malnutrition in Malawi; Ann.trop.paed; 20:1, 1 March 2000, pp. 50-56(7)

[10] Hanifa Bachou; Severe malnutrition with and without HIV-1 infection in hospitalized children in Kampala, Uganda; Nutrition Journal 2006. 5:27

[11] London Peter Moszynski; AIDS and malnutrition inextricably Linked; BMJ 2006; 333:64 (8 July).

[12] Schewenk A, Buger B, Wessel D, Stutzer H, Ziegenhagen D, 
Diehl V, Schrape M; Clinical risk factors for malnutrition in HIV-1-infected patients; AIDS. 1993, 7: 1213-1219.

[13] NI Paton, S Sangeetha, A Earnest and R Bellamy; The impact of malnutrition on survival \& the CD4 count response in HIV-infected Patients starting ART; HIV Medicine 2006, 7, $323-330$

[14] WHO, Regional Office for the Western Pacific; HEALTH RESEARCH METHODOLOGY, A Guide for Training in Research Methods; Second Edition; Manila, 2001.

[15] Neil Pearce ; A Short Introduction to Epidemiology; Second Edition; 2005.

[16] Kenneth F Schulz, David A Grimes; Epidemiology series; The Lancet; 2002; 359:9303: 341-345.

[17] WHO, Management of severe malnutrition: a manual for physicians and other senior health workers, Geneva 1999.

[18] WHO, WHO case definitions of HIV for surveillance and revised clinical staging and immunological classification of HIV-related disease in adults and children, 2006, GENEVA.
[19] Buford Nichols; Treatment of severe malnutrition in developing countries, Uptodate version 15.3

[20] Rebecca T Kirkland; Etiology and evaluation of failure to thrive in children younger than two years; Uptodate version 15.3

[21] Tindyebwa D, Kayita J, Mosoke P, et al, eds. 2006. Handbook on Paediatric AIDS in Africa. Uganda: African Network for the Care of Children Affected by AIDS, ANNECA.

[22] Michael Golden; The management of severe acute malnutrition; A manual for Ethiopia; UNICEF, 2002.

[23] Fawzi WW, Msamanga GI, Spiegelman D et al. (2004) "Randomized Trial of Multivitamin Supplements and HIV Disease Progression and Mortality" New England Journal of Medicine 351:23-32.

[24] Shorr IJ. How to weigh and measure children: assessing the nutritional status of young children in household surveys. New York: Department of Technical Co-operation for Development and Statistical Office, United Nations, 1986. 